\title{
INEXACT NEWTON REGULARIZATION USING CONJUGATE GRADIENTS AS INNER ITERATION*
}

\author{
ANDREAS RIEDER ${ }^{\dagger}$
}

\begin{abstract}
In our papers [Inverse Problems, 15 (1999), pp. 309-327] and [Numer. Math., 88 (2001), pp. 347-365] we proposed algorithm REGINN, an inexact Newton iteration for the stable solution of nonlinear ill-posed problems. REGINN consists of two components: the outer iteration, which is a Newton iteration stopped by the discrepancy principle, and an inner iteration, which computes the Newton correction by solving the linearized system. The convergence analysis presented in both papers covers virtually any linear regularization method as inner iteration, especially Landweber iteration, $\nu$-methods, and Tikhonov-Phillips regularization. In the present paper we prove convergence rates for REGINN when the conjugate gradient method, which is nonlinear, serves as inner iteration. Thereby we add to a convergence analysis of Hanke, who had previously investigated REGINN furnished with the conjugate gradient method [Numer. Funct. Anal. Optim., 18 (1997), pp. 971-993]. By numerical experiments we illustrate that the conjugate gradient method outperforms the $\nu$-method as inner iteration.
\end{abstract}

Key words. nonlinear ill-posed problems, inexact Newton iteration, conjugate gradients, $\nu$ methods, regularization

AMS subject classifications. $65 \mathrm{~J} 20,65 \mathrm{~J} 22$

DOI. $10.1137 / 040604029$

1. Introduction. Our goal is to find a stable approximate solution of the nonlinear ill-posed problem

$$
F(x)=y^{\delta}
$$

where $F: \mathrm{D}(F) \subset X \rightarrow Y$ operates between the Hilbert spaces $X$ and $Y$. Here, $\mathrm{D}(F)$ denotes the domain of definition of $F$, and $y^{\delta}$ is a noisy version of the exact but unknown data $y=F\left(x^{+}\right)$satisfying

$$
\left\|y-y^{\delta}\right\|_{Y} \leq \delta .
$$

The nonnegative noise level $\delta$ is assumed to be known.

In $[10,11]$ we proposed algorithm REGINN for solving (1.1). As a Newton-type algorithm, REGINN updates the actual iterate $x_{n}$ by adding a correction step $s_{n}^{\delta}$ obtained from solving a linearization of (1.1):

$$
x_{n+1}=x_{n}+s_{n}^{\delta}, \quad n \in \mathbb{N}_{0},
$$

with an initial guess $x_{0}$. For obvious reasons we like to have $s_{n}^{\delta}$ as close as possible to the exact Newton step

$$
s_{n}^{\mathrm{e}}=x^{+}-x_{n} .
$$

* Received by the editors August 2, 2004; accepted for publication (in revised form) September 27, 2004; published electronically June 30, 2005.

http://www.siam.org/journals/sinum/43-2/60402.html

${ }^{\dagger}$ Institut für Praktische Mathematik und Institut für Wissenschaftliches Rechnen und Mathematische Modellbildung, Universität Karlsruhe, 76128 Karlsruhe, Germany (andreas.rieder@math. uni-karlsruhe.de, www.mathematik.uni-karlsruhe.de/ rieder). 
Assuming $F$ to be Fréchet differentiable with derivative $F^{\prime}: \mathrm{D}(F) \rightarrow \mathcal{L}(X, Y)$, the exact Newton step satisfies the linear equation

$$
F^{\prime}\left(x_{n}\right) s_{n}^{\mathrm{e}}=y-F\left(x_{n}\right)-E\left(x^{+}, x_{n}\right)=: b_{n},
$$

where $E(v, w):=F(v)-F(w)-F^{\prime}(w)(v-w)$ is the linearization error.

Unfortunately, the above right-hand side $b_{n}$ is not available; however, we know a perturbed version

$$
b_{n}^{\varepsilon}:=y^{\delta}-F\left(x_{n}\right) \quad \text { with }\left\|b_{n}-b_{n}^{\varepsilon}\right\|_{Y} \leq \delta+\left\|E\left(x^{+}, x_{n}\right)\right\|_{Y} .
$$

Therefore, we determine the correction step $s_{n}^{\delta}$ as a solution of

$$
F^{\prime}\left(x_{n}\right) s=b_{n}^{\varepsilon} .
$$

Here, we have to take into account that the ill-posedness of (1.1) is passed on to (1.4). For instance, if $F$ is completely continuous, then $F^{\prime}\left(x_{n}\right)$ is a compact operator (see, e.g., Zeidler [13, Proposition 7.33]); hence, (1.4) is ill-posed.

Depending on how $s_{n}^{\delta}$ is stably obtained from (1.4), different methods arise, for instance, the nonlinear Landweber method (Hanke, Neubauer, and Scherzer [6]), the Gauß-Newton method (see, e.g., Bakushinskii [1] and Kaltenbacher [7]), and the Levenberg-Marquardt scheme (Hanke [4]).

In the next few lines we recall briefly how REGINN works. First, a regularization scheme is applied to the linear system (1.4), obtaining

$$
s_{n, r}:=g_{r}\left(A_{n}^{*} A_{n}\right) A_{n}^{*} b_{n}^{\varepsilon},
$$

where $A_{n}=F^{\prime}\left(x_{n}\right)$ and $g_{r}:\left[0,\left\|A_{n}\right\|^{2}\right] \rightarrow \mathbb{R}$ is the piecewise continuous filter function of the chosen regularization method. The parameter $r \in \mathbb{N}$ is called the regularization parameter. For instance, the filter functions belonging to the Tikhonov-Phillips regularization, the Landweber iteration, and the $\nu$-methods are explicitly known; see, e.g., $[2,12]$, where more examples can be found. The filter functions $g_{r}$ of both latter examples are polynomials of degree $r-1$. The conjugate gradients method (cg-method) can also be described by filter polynomials $g_{r}$ of degree $r-1$, which, however, do depend on the right-hand side $b_{n}^{\varepsilon}: g_{r}(\cdot)=g_{r}\left(\cdot, b_{n}^{\varepsilon}\right)$. Therefore, the cg-method is a nonlinear scheme in contrast to the other mentioned examples.

Now we have to select a regularization parameter $r_{n}$. In REGINN $r_{n}$ is picked as the smallest number at which the relative (linear) residual is smaller than a given tolerance $\left.\left.\mu_{n} \in\right] 0,1\right]$, that is,

$$
\left\|A_{n} s_{n, r_{n}}-b_{n}^{\varepsilon}\right\|_{Y}<\mu_{n}\left\|b_{n}^{\varepsilon}\right\|_{Y} \leq\left\|A_{n} s_{n, i}-b_{n}^{\varepsilon}\right\|_{Y}, \quad i=1, \ldots, r_{n}-1 .
$$

The tolerances should not be too small to guarantee existence of $r_{n}$; see Lemma 2.1 below. A meaningful strategy to adapt the $\mu_{n}$ 's dynamically was proposed in [10]. Setting $s_{n}^{\delta}:=s_{n, r_{n}}$ we end up with the Newton iteration

$$
x_{n+1}=x_{n}+g_{r_{n}}\left(A_{n}^{*} A_{n}\right) A_{n}^{*} b_{n}^{\varepsilon}, \quad n \in \mathbb{N}_{0},
$$

which has to be stopped in time to avoid noise amplification. A well-established stopping rule is the discrepancy principle: Choose $R>0$ and accept iterate $x_{N}$ as an approximate solution of (1.1) that fulfills

$$
\left\|y^{\delta}-F\left(x_{N}\right)\right\|_{Y} \leq R \delta<\left\|y^{\delta}-F\left(x_{k}\right)\right\|_{Y}, \quad k=0, \ldots, N-1 .
$$




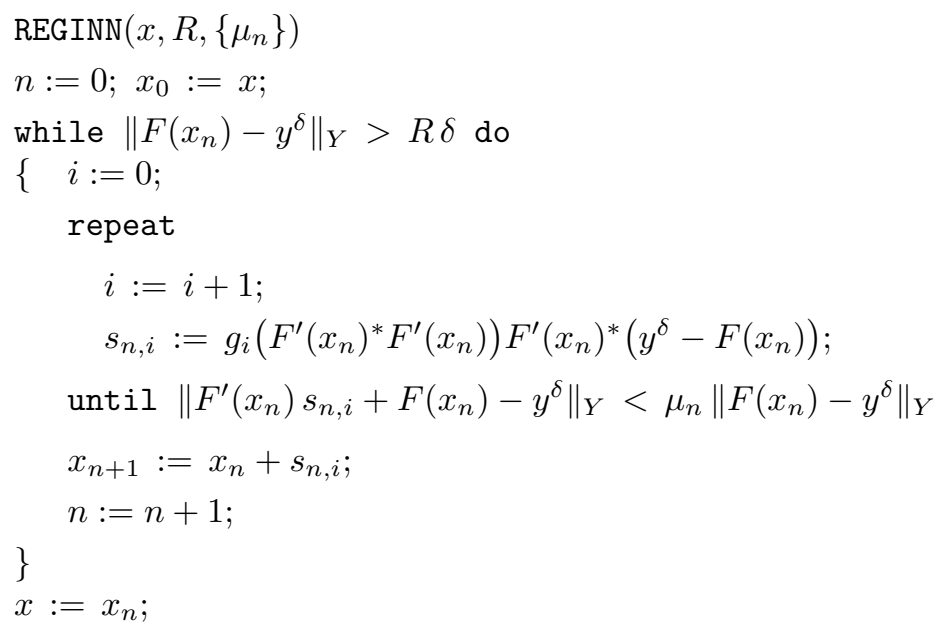

FIG. 1.1. Algorithmic realization of REGINN (

For an algorithmic realization of REGINN, see Figure 1.1. The inner repeat-loop provides the Newton update $s_{n, r_{n}}$ and the outer while-loop implements the Newton iteration stopped by the discrepancy principle.

In [11] we were able to verify (under reasonable assumptions) that REGINN with a linear regularization scheme $\left\{g_{r}\right\}_{r \in \mathbb{N}}$ is well defined and indeed terminates. Moreover, we proved the existence of a positive $\kappa_{\min }<1$ such that the source condition ${ }^{1}$

$$
\left.\left.x^{+}-x_{0} \in \mathrm{R}\left(\left|F^{\prime}\left(x^{+}\right)\right|^{\kappa}\right) \quad \text { for a } \kappa \in\right] \kappa_{\min }, 1\right]
$$

implies the suboptimal convergence rate $^{2}$

$$
\left\|x^{+}-x_{N(\delta)}\right\|_{X}=\mathrm{O}\left(\delta^{\left(\kappa-\kappa_{\min }\right) /(1+\kappa)}\right) \quad \text { as } \delta \rightarrow 0 .
$$

In the present paper we will improve upon the convergence results for REGINN: We will verify that (1.7) implies (1.8) even when the cg-method serves as inner iteration of REGINN. Thus we supplement a convergence analysis of Hanke [5], who had previously investigated REGINN with the cg-method as inner iteration: Under a slightly weaker version of our general assumption on the nonlinearity (see (2.1) below), Hanke proved convergence of $\left\{x_{N(\delta)}\right\}_{\delta>0}$ to a set of solutions of $F(x)=y$ as $\delta \rightarrow 0$.

This paper is structured as follows. In the next two sections we compile facts about REGINN and the cg-method which we will need later on in our analysis. In section 4 we show that REGINN is well defined under (1.7) and terminates with an approximation to $x^{+}$. Then the regularization property (1.8) will be verified (section 5). Finally, we present numerical experiments for a parameter identification model problem and end with concluding remarks in section 7. Some lengthy and technical proofs from sections 3 and 4 are shifted to Appendices A and B, respectively.

\footnotetext{
${ }^{1} \mathrm{By} \mathrm{R}(B)$ we denote the range of the operator $B$, and $|B|$ is the square root of $B^{*} B$.

${ }^{2}$ For linear inverse problems $A x=y^{\delta}$ the regularization error cannot decrease faster than $\mathrm{O}\left(\delta^{\kappa /(1+\kappa)}\right)$ as $\delta \rightarrow 0$ under the source condition $x^{+}-x_{0} \in \mathrm{R}\left(|A|^{\kappa}\right)$ in general; see, e.g., [2, section 3.2] or [12, Kapitel 3.2.3]. Regularization schemes attaining the maximal order are therefore called order-optimal.
} 
2. General assumptions and termination of the repeat-loop. Throughout the paper we assume $F: \mathrm{D}(F) \subset X \rightarrow Y$ to be continuously Fréchet differentiable with derivative $F^{\prime}: \mathrm{D}(F) \rightarrow \mathcal{L}(X, Y)$. Moreover, let $x^{+} \in \mathrm{D}(F), y=F\left(x^{+}\right), y^{\delta} \in Y$ with $\left\|y-y^{\delta}\right\|_{Y} \leq \delta, A=F^{\prime}\left(x^{+}\right)$, and $A_{n}=F\left(x_{n}\right)$.

Our analysis relies heavily on the local factorization (2.1) of $F^{\prime}:$ Let $Q: X \times X \rightarrow$ $\mathcal{L}(X, Y)$ be a mapping such that

$$
F^{\prime}(v)=Q(v, w) F^{\prime}(w) \quad \text { with }\|I-Q(v, w)\| \leq C_{Q}\|v-w\|_{X}
$$

for all $v, w \in B_{\rho}\left(x^{+}\right) \subset \mathrm{D}(F)$, the open ball of radius $\rho$ about $x^{+}$. Here, $C_{Q}$ is a positive constant. For a discussion of the nontrivial factorization (2.1) and for examples of meaningful operators satisfying $(2.1)$, we refer to [6, 10, 11], [12, Kapitel 7.3], and the literature cited therein.

Let $C_{Q} \rho<1$. Then (2.1) gives

$$
\|F(v)-F(w)\|_{Y} \geq\left(1-C_{Q} \rho\right)\left\|F^{\prime}(w)(v-w)\right\|_{Y}
$$

as well as

$$
\|E(v, w)\|_{Y} \leq \omega\|F(v)-F(w)\|_{Y} \quad \text { for all } v, w \in B_{\rho}\left(x^{+}\right),
$$

where $\omega:=C_{Q} \rho /\left(1-C_{Q} \rho\right)$; see [10, section 3] or [12, Lemma 7.3.9]. Observe that $\omega<1$ for $C_{Q} \rho<1 / 2$.

In our subsequent analysis we will frequently use the following estimate: For $x, y \in B_{\rho}\left(x^{+}\right)$and $C_{Q} \rho<1 / 2$ we have

$$
\left\|\left|F^{\prime}(x)\right|^{-\kappa}\left|F^{\prime}(y)\right|^{\kappa}\right\| \leq\left(1-2 C_{Q} \rho\right)^{-\kappa}=: C_{K, \kappa} \quad \text { for all } \kappa \in[0,1],
$$

which is due to Kaltenbacher [7, Lemma 2.2]; see also [12, Lemma 7.5.16].

Using (2.3) we will bound the data error $\left\|b_{n}^{\varepsilon}-b_{n}\right\|_{Y}$ in terms of $\delta, \omega$, and the nonlinear defect

$$
d_{n}:=\left\|y^{\delta}-F\left(x_{n}\right)\right\|_{Y}=\left\|b_{n}^{\varepsilon}\right\|_{Y} .
$$

For $x_{n} \in B_{\rho}\left(x^{+}\right)$we find

$$
\left\|b_{n}^{\varepsilon}-b_{n}\right\|_{Y} \leq(1+\omega) \delta+\omega d_{n}:=\varepsilon=\varepsilon\left(x_{n}, \delta\right) .
$$

We recall a result from [10] which gives conditions on $\mu_{n}$ to stop the repeat-loop.

LEMma 2.1. Let $\left\{g_{r}\right\}_{r \in \mathbb{N}}$ be the filter function of a linear or nonlinear regularization scheme for which the discrepancy principle returns a well-defined stopping index; that is, for $\tau>1$ there exists a smallest index $r_{S}$ with $\left\|A_{n} s_{n, r_{S}}-b_{n}^{\varepsilon}\right\|_{Y} \leq \tau \varepsilon$. Further let (2.1) hold true with $C_{Q} \rho<1 / 2$ and assume $x_{n} \in B_{\rho}\left(x^{+}\right)$, where $n<N$. If $R \geq(1+\omega) /(1-\omega)$, then the repeat-loop of algorithm REGINN terminates for any

$$
\left.\left.\mu_{n} \in\right] \omega+\frac{(1+\omega) \delta}{d_{n}}, 1\right]
$$

The lower bound on $R$ in Lemma 2.1 guarantees that the interval for $\mu_{n}$ is nonempty. Since $b_{n} \in \mathrm{R}\left(A_{n}\right)$ (see (1.3)), all regularization methods mentioned in section 1 (Tikhonov-Phillips, Landweber, $\nu$-method, cg-method) satisfy the requirement of Lemma 2.1; see, e.g., Engl, Hanke, and Neubauer [2, Chapter 4.3] or [12, Kapitel 3.4]. 
3. The method of conjugate gradients: Preliminaries. Here we recall some basic facts of the cg-method which we will need later in the paper. More details as well as all proofs can be found in, e.g., Engl, Hanke, and Neubauer [2, Chapter 7] or [12, Kapitel 5.3].

Let $T \in \mathcal{L}(X, Y)$ and $\boldsymbol{\eta} \in Y$. The cg-method is an iteration for solving the normal equation $T^{*} T \boldsymbol{\zeta}=T^{*} \boldsymbol{\eta}$. Starting with $\boldsymbol{\xi}_{0} \in X$ the cg-method produces a sequence $\left\{\boldsymbol{\xi}_{m}\right\}_{m \in \mathbb{N}_{0}}$ with the minimization property

$$
\left\|\boldsymbol{\eta}-T \boldsymbol{\xi}_{m}\right\|_{Y}=\min \left\{\|\boldsymbol{\eta}-T \boldsymbol{\xi}\|_{Y} \mid \boldsymbol{\xi} \in X, \boldsymbol{\xi}-\boldsymbol{\xi}_{0} \in U_{m}\right\}, \quad m \geq 1,
$$

where $U_{m}$ is the $m$ th Krylov space,

$$
U_{m}:=\operatorname{span}\left\{T^{*} r^{0},\left(T^{*} T\right) T^{*} r^{0},\left(T^{*} T\right)^{2} T^{*} r^{0}, \ldots,\left(T^{*} T\right)^{m-1} T^{*} r^{0}\right\}
$$

with $r^{0}:=\boldsymbol{\eta}-T \boldsymbol{\xi}_{0}$. Therefore, $\boldsymbol{\xi}_{m}, m \geq 1$, can be expressed by

$$
\boldsymbol{\xi}_{m}=\boldsymbol{\xi}_{0}+q_{m-1}\left(T^{*} T\right) T^{*}\left(\boldsymbol{\eta}-T \boldsymbol{\xi}_{0}\right)
$$

with a polynomial $q_{m-1}$ of degree $m-1$. Closely related to $q_{m-1}$ is the residual polynomial $p_{m}(\lambda)=1-\lambda q_{m-1}(\lambda)$ of degree $m$ satisfying

$$
\boldsymbol{\eta}-T \boldsymbol{\xi}_{m}=p_{m}\left(T T^{*}\right)\left(\boldsymbol{\eta}-T \boldsymbol{\xi}_{0}\right) .
$$

Both polynomials depend on $\boldsymbol{\eta}$ : $q_{m-1}(\cdot)=q_{m-1}(\cdot, \boldsymbol{\eta})$ and $p_{m}(\cdot)=p_{m}(\cdot, \boldsymbol{\eta})$. As soon as $T^{*}\left(\boldsymbol{\eta}-T \boldsymbol{\xi}_{k}\right)=0$ holds true, the cg-sequence is finite, that is, $\boldsymbol{\xi}_{m}=\boldsymbol{\xi}_{k}$ for all $m \geq k$. Accordingly,

$$
\boldsymbol{m}_{\mathrm{T}}:=\sup \left\{m \in \mathbb{N} \mid T^{*}\left(\boldsymbol{\eta}-T \boldsymbol{\xi}_{m-1}\right) \neq 0\right\}
$$

is called the ultimate termination index of the cg-method $\left(\boldsymbol{m}_{\mathrm{T}}=\infty\right.$ is allowed and the supremum of the empty set is understood as zero).

The residual polynomials are orthogonal with respect to the inner product $\langle\varphi, \psi\rangle_{\Pi}$ $:=\left\langle\varphi\left(T^{*} T\right) T^{*} \boldsymbol{\eta}, \psi\left(T^{*} T\right) T^{*} \boldsymbol{\eta}\right\rangle_{X}$, which is defined on the space of all polynomials:

$$
\left\langle p_{i}, p_{j}\right\rangle_{\Pi}=0 \text { for all } 1 \leq i, j \leq \boldsymbol{m}_{\mathrm{T}} \text { with } i \neq j .
$$

The orthogonality of $\left\{p_{m}\right\}_{1 \leq m \leq m_{\mathrm{T}}}$ has several consequences. The residual polynomials satisfy a three-term recursion which can be used to compute $\boldsymbol{\xi}_{m}$ iteratively from $\boldsymbol{\xi}_{m-1}$ in a rather cheap way; see Figure 3.1. Moreover, $p_{m}$ has $m$ simple roots $\left.\lambda_{m, j} \in\right] 0,\|T\|^{2}[, j=1, \ldots, m$, which we order by

$$
0<\lambda_{m, 1}<\lambda_{m, 2}<\cdots<\lambda_{m, m}<\|A\|^{2} .
$$

Because of its normalization $p_{m}(0)=1, p_{m}$ decomposes into the following linear factors:

$$
p_{m}(\lambda)=\prod_{j=1}^{m}\left(1-\lambda / \lambda_{m, j}\right)=\prod_{j=1}^{m} \frac{\lambda_{m, j}-\lambda}{\lambda_{m, j}} .
$$

Although we know neither $q_{m-1}$ nor $p_{m}$ explicitly, some useful information about both polynomials is available.

Lemma 3.1. For $0<\Lambda \leq \lambda_{m, 1}$ and $1 \leq m \leq m_{\mathrm{T}}$, we have that

$$
\sup _{0 \leq \lambda \leq \Lambda}\left|q_{m-1}(\lambda, \boldsymbol{\eta})\right|=q_{m-1}(0, \boldsymbol{\eta})=-p_{m}^{\prime}(0, \boldsymbol{\eta})=\sum_{j=1}^{m} \lambda_{m, j}^{-1} .
$$


cg-algorithm for $T \in \mathcal{L}(X, Y), \boldsymbol{\eta} \in Y$ and starting guess $\boldsymbol{\xi}_{0} \in X$.

$$
\begin{aligned}
& r^{0}:=\boldsymbol{\eta}-T \boldsymbol{\xi}_{0} ; p^{1}=a^{0}:=T^{*} r^{0} ; \\
& m:=1 \text {; } \\
& \text { while }\left(a^{m-1} \neq 0\right) \\
& \left\{q^{m}:=T p^{m}\right. \text {; } \\
& \alpha_{m}:=\left\|a^{m-1}\right\|_{X}^{2} /\left\|q^{m}\right\|_{Y}^{2} ; \\
& \boldsymbol{\xi}_{m}:=\boldsymbol{\xi}_{m-1}+\alpha_{m} p^{m} ; \\
& r^{m}:=r^{m-1}-\alpha_{m} q^{m} ; \\
& a^{m}:=T^{*} r^{m} ; \\
& \beta_{m}:=\left\|a^{m}\right\|_{X}^{2} /\left\|a^{m-1}\right\|_{X}^{2} ; \\
& p^{m+1}:=a^{m}+\beta_{m} p^{m} ; \\
& m:=m+1 ;\}
\end{aligned}
$$

FIG. 3.1. Conjugate gradients algorithm.

The next result is proved in Appendix A and will be used twice in our convergence analysis of REGINN with the cg-method as inner iteration.

Lemma 3.2. Let $\left\{\boldsymbol{\xi}_{m}\right\}_{0 \leq m \leq m_{\mathrm{T}}}, \boldsymbol{\xi}_{0}=0$, be the cg-sequence with respect to $T \in$ $\mathcal{L}(X, Y)$ and $\boldsymbol{\eta} \in Y$. Further, let $\boldsymbol{\xi}$ be in $\mathrm{D}\left(|T|^{-\mu}\right)$ for a $\mu \in[0,1]$. Then, for any $\nu \in[0, \mu]$, we have that

$$
\begin{gathered}
\left\||T|^{-\nu}\left(\boldsymbol{\xi}_{m}-\boldsymbol{\xi}\right)\right\|_{X} \leq q_{m-1}(0, \boldsymbol{\eta})^{(\nu+1) / 2}\left(\left\|T\left(\boldsymbol{\xi}_{m}-\boldsymbol{\xi}\right)\right\|_{Y}+\|\boldsymbol{\eta}-T \boldsymbol{\xi}\|_{Y}\right) \\
+q_{m-1}(0, \boldsymbol{\eta})^{(\nu-\mu) / 2}\left\||T|^{-\mu} \boldsymbol{\xi}\right\|_{X} .
\end{gathered}
$$

4. Termination of REGINN with conjugate gradients. The convergence of REGINN will be established by bounding the Newton corrections $s_{n, r_{n}}$ sharply enough. Indeed, we will show that the Newton corrections decrease geometrically in $n$. Thus, the Newton iterates stay in a ball about $x_{0}$.

Recall the assumptions and notation from section 2 and let the cg-method be the inner iteration of REGINN exclusively throughout this section.

Lemma 4.1. Suppose $s_{n, r_{n}}$ is well defined. Then

$$
\left\|s_{n, r_{n}}\right\|_{X}<3 q_{r_{n}-1}\left(0, b_{n}^{\varepsilon}\right)^{1 / 2} d_{n} .
$$

Proof. We apply Lemma 3.2 with $T=A_{n}, \mu=\nu=0, \boldsymbol{\xi}=0, \boldsymbol{\eta}=b_{n}^{\varepsilon}, \boldsymbol{\xi}_{m}=$ $s_{n, r_{n}}=q_{r_{n}-1}\left(A_{n}^{*} A, b_{n}^{\varepsilon}\right) A_{n}^{*} b_{n}^{\varepsilon}$, that is, $m=r_{n}$. Thus,

$$
\left\|s_{n, r_{n}}\right\|_{X} \leq q_{r_{n}-1}\left(0, b_{n}^{\varepsilon}\right)^{1 / 2}\left(\left\|A_{n} s_{n, r_{n}}\right\|_{Y}+\left\|b_{n}^{\varepsilon}\right\|_{Y}\right) .
$$

We are done by $\left\|A_{n} s_{n, r_{n}}\right\|_{Y} \leq\left\|A_{n} s_{n, r_{n}}-b_{n}^{\varepsilon}\right\|_{Y}+\left\|b_{n}^{\varepsilon}\right\|_{Y} \leq\left(\mu_{n}+1\right)\left\|b_{n}^{\varepsilon}\right\|_{Y}$.

In the following we bound each of the factors on the right-hand side of (4.1). From [10, Lemma 4.1] (see also [12, Lemma 7.5.9]) we already know that the nonlinear residuals $d_{n}$ decrease linearly.

LEMma 4.2. Suppose that the nth iterate $x_{n}$ of REGINN is well defined and lies in $B_{\rho}\left(x^{+}\right)$. Further, let (2.3) hold true with

$$
\omega<\eta /(2+\eta) \text { for one } \eta<1 .^{3}
$$

\footnotetext{
${ }^{3}$ This restriction is satisfied, for instance, if (2.1) holds true and $\rho$ is small enough.
} 
If, moreover,

$$
\left.\left.R \geq \frac{1+\omega}{\eta-(2+\eta) \omega} \quad \text { and } \quad \mu_{n} \in\right] \omega+\frac{(1+\omega) \delta}{d_{n}}, \eta-(1+\eta) \omega\right]
$$

as well as $x_{n+1} \in B_{\rho}\left(x^{+}\right)$, then

$$
\frac{d_{n+1}}{d_{n}}=\frac{\left\|y^{\delta}-F\left(x_{n+1}\right)\right\|_{Y}}{\left\|y^{\delta}-F\left(x_{n}\right)\right\|_{Y}}<\frac{\mu_{n}+\omega}{1-\omega} \leq \eta
$$

4.1. Bounding $\boldsymbol{q}_{\boldsymbol{r}_{n-1}}\left(\mathbf{0}, \boldsymbol{b}_{\boldsymbol{n}}^{\varepsilon}\right)$. We assume the existence of $w \in X$ and $\kappa \in[0,1]$ such that

$$
s_{0}^{\mathrm{e}}=x^{+}-x_{0}=|A|^{\kappa} w
$$

where $A=F^{\prime}\left(x^{+}\right)$. To formulate the bound for $q_{r_{k}-1}\left(0, b_{k}^{\varepsilon}\right)$ we introduce the ratio

$$
\tau_{k}:=\mu_{k} d_{k} / \varepsilon\left(x_{k}, \delta\right)
$$

which is greater than 1 under the hypotheses of Lemma 2.1.

Lemma 4.3. Let (2.1) hold true with $C_{Q} \rho<1 / 2$ (thus, $\omega<1$ in (2.3)) and assume that the first $n<N$ iterates $\left\{x_{1}, \ldots, x_{n}\right\}$ of REGINN exist and stay in $B_{\rho}\left(x^{+}\right)$. Further, let $x_{0} \in B_{\rho}\left(x^{+}\right)$satisfy the source condition (4.2).

Then $s_{k}^{\mathrm{e}}=x^{+}-x_{k} \in \mathrm{D}\left(\left|A_{k}\right|^{-\kappa}\right), 0 \leq k \leq n$. Moreover, if $R \geq(1+\omega) /(1-\omega)$ and if $\left.\left.\mu_{k} \in\right] \omega+(1+\omega) \delta / d_{k}, 1\right], 0 \leq k \leq n$, then for any $\left.\Theta \in\right] 0,1[$ such that $\Theta \min \left\{\tau_{0}, \ldots, \tau_{n}\right\}>1$ we have

$$
q_{r_{k}-1}\left(0, b_{k}^{\varepsilon}\right)^{(\kappa+1) / 2} \leq \frac{a_{\Theta}}{\Theta \tau_{k}-1} \varepsilon\left(x_{k}, \delta\right)^{-1}\left\|\left|A_{k}\right|^{-\kappa} s_{k}^{\mathrm{e}}\right\|_{X}, \quad 0 \leq k \leq n,
$$

where $a_{\Theta}$ is a positive constant depending only on $\Theta$ and $\kappa$.

Proof. See Appendix B for the proof.

Let us summarize what we found so far. Starting from (4.1) we are able to bound the Newton steps under the assumptions of Lemma 4.3 by

$$
\left\|s_{k, r_{k}}\right\|_{X}<3\left(\frac{a_{\Theta}}{\Theta \tau_{k}-1}\right)^{1 /(\kappa+1)} \varepsilon\left(x_{k}, \delta\right)^{-1 /(\kappa+1)}\left\|\left|A_{k}\right|^{-\kappa} s_{k}^{\mathrm{e}}\right\|_{X}^{1 /(\kappa+1)} d_{k} .
$$

4.2. Bounding $\left\|\left|\boldsymbol{A}_{\boldsymbol{k}}\right|^{-\kappa} s_{\boldsymbol{k}}^{\mathrm{e}}\right\|_{\boldsymbol{X}}$. Before we are able to establish termination of REGINN by (4.4), we have to know how $\left\|\left|A_{k}\right|^{-\kappa} s_{k}^{\mathrm{e}}\right\|_{X}$ behaves in $k$. Since $s_{k}^{\mathrm{e}}=x^{+}-$ $x_{k}=x^{+}-x_{k-1}-s_{k-1, r_{k-1}}=s_{k-1}^{\mathrm{e}}-s_{k-1, r_{k-1}}$ we conclude that

$$
\begin{aligned}
\left\|\left|A_{k}\right|^{-\kappa} s_{k}^{\mathrm{e}}\right\|_{X} & =\left\|\left|A_{k}\right|^{-\kappa}\left(s_{k-1}^{\mathrm{e}}-s_{k-1, r_{k-1}}\right)\right\|_{X} \\
& \stackrel{(2.4)}{\leq} C_{K, \kappa}\left\|\left|A_{k-1}\right|^{-\kappa}\left(s_{k-1}^{\mathrm{e}}-s_{k-1, r_{k-1}}\right)\right\|_{X} .
\end{aligned}
$$


We estimate the norm on the right by applying Lemma 3.2 with $T=A_{k-1}, \mu=\nu=\kappa$, $\boldsymbol{\xi}=s_{k-1}^{\mathrm{e}} \boldsymbol{\eta}=b_{k-1}^{\varepsilon}$, and $\boldsymbol{\xi}_{m}=s_{k-1, r_{k-1}}$. Hence,

$$
\begin{aligned}
& \left\|\left|A_{k}\right|^{-\kappa} s_{k}^{\mathrm{e}}\right\|_{X} \leq C_{K, \kappa} q_{r_{k-1}-1}\left(0, b_{k-1}^{\varepsilon}\right)^{(\kappa+1) / 2}\left(\left\|A_{k-1} s_{k-1, r_{k-1}}-b_{k-1}\right\|_{Y}\right. \\
& \left.+\left\|b_{k-1}^{\varepsilon}-b_{k-1}\right\|_{Y}\right)+\left\|\left|A_{k-1}\right|^{-\kappa} s_{k-1}^{\mathrm{e}}\right\|_{X} \\
& \leq C_{K, \kappa} q_{r_{k-1}-1}\left(0, b_{k-1}^{\varepsilon}\right)^{(\kappa+1) / 2}(\underbrace{\mu_{k-1} d_{k-1}}_{\left(\stackrel{4.3)}{=} \tau_{k-1} \varepsilon\left(x_{k-1}, \delta\right)\right.}+2 \varepsilon\left(x_{k-1}, \delta\right)) \\
& +||\left|A_{k-1}\right|^{-\kappa} s_{k-1}^{\mathrm{e}} \|_{X} \\
& \leq 3 C_{K, \kappa} q_{r_{k-1}-1}\left(0, b_{k-1}^{\varepsilon}\right)^{(\kappa+1) / 2} \tau_{k-1} \varepsilon\left(x_{k-1}, \delta\right) \\
& +||\left|A_{k-1}\right|^{-\kappa} s_{k-1}^{\mathrm{e}} \|_{X} \\
& \leq\left(3 C_{K, \kappa} a_{\Theta} \frac{\tau_{k-1}}{\Theta \tau_{k-1}-1}+1\right)\left\|\left|A_{k-1}\right|^{-\kappa} s_{k-1}^{\mathrm{e}}\right\|_{X},
\end{aligned}
$$

where we used Lemma 4.3 in the last step. Inductively, we end up with the following lemma.

Lemma 4.4. Let (2.1) hold true with $C_{Q} \rho<1 / 2$ (thus, $\omega<1$ in (2.3)) and assume that the first $n<N$ iterates $\left\{x_{1}, \ldots, x_{n}\right\}$ of REGINN exist and stay in $B_{\rho}\left(x^{+}\right)$. Further, choose $R \geq(1+\omega) /(1-\omega)$ and let $x_{0} \in B_{\rho}\left(x^{+}\right)$satisfy the source condition (4.2).

If $\left.\left.\mu_{k} \in\right] \omega+(1+\omega) \delta / d_{k}, 1\right], 0 \leq k \leq n$, and if $\left.\Theta \in\right] 0,1\left[\right.$ is such that $\Theta \tau_{k}>1$, $0 \leq k \leq n$, then

$$
\left\|\left|A_{k}\right|^{-\kappa} s_{k}^{\mathrm{e}}\right\|_{X} \leq C_{K, \kappa} \Lambda_{n}^{k}\|w\|_{X} \quad \text { for } k=0, \ldots, n
$$

with

$$
\Lambda_{n}=1+3 C_{K, \kappa} a_{\Theta} \frac{t_{n}}{\Theta t_{n}-1} \quad \text { and } \quad t_{n}=\min \left\{\tau_{0}, \ldots, \tau_{n}\right\} .
$$

4.3. Termination. We are now able to verify termination of REGINN with conjugate gradients as inner iteration: under reasonable technical assumptions all iterates remain in $B_{\rho}\left(x_{0}\right)$ and REGINN delivers an approximation $x_{N(\delta)}$ to $x^{+}$. The following theorem is the counterpart of Theorem 3.3 from [11] (see also [12, Satz 7.5.14]) and will be proved along the same lines.

THEOREM 4.5. Let (2.1) hold true with $C_{Q} \rho<1 / 2$ (thus, $\omega<1$ in (2.3)). Let $\tau>1$ and let $\Theta \in] 0,1[$ be such that $\Theta \tau>1$. Set

$$
\Lambda=1+3 C_{K, \kappa} a_{\Theta} \frac{\tau}{\Theta \tau-1},
$$

where $C_{K, \kappa}=\left(1-C_{Q} \rho\right)^{-\kappa}$ (see (2.4)), and $a_{\Theta}$ comes from the estimate in Lemma 4.3. Suppose that (2.3) is satisfied with

$$
\omega<\frac{\eta}{\eta+\tau+1}, \quad \text { where } \eta \Lambda<1 .^{4}
$$

Assume that the starting guess $x_{0} \in B_{\rho / 2}\left(x^{+}\right)$is chosen such that the source condition (4.2) applies for $\kappa$ restricted to $\left.] \log _{1 / \eta} \Lambda, 1\right]$ and that the product $\|w\|_{X} \| y-$

\footnotetext{
${ }^{4}$ This restriction is satisfied, for instance, if (2.1) holds true and $\rho$ is small enough.
} 
$F\left(x_{0}\right) \|_{Y}^{\kappa}$ is sufficiently small. If

$$
R \geq \frac{\tau(1+\omega)}{\eta-\omega(\eta+\tau+1)} \quad \text { and } \quad \mu_{k} \in\left[\tau\left(\omega+\frac{(1+\omega) \delta}{d_{k}}\right), \eta-(1+\eta) \omega\right]
$$

for $k \geq 0$, then there is an $N(\delta) \in \mathbb{N}$ and a $\bar{\delta}>0$ such that all iterates $\left\{x_{1}, \ldots, x_{N(\delta)}\right\}$ are well defined and stay in $B_{\rho}\left(x^{+}\right)$for all noise levels $\left.\left.\delta \in\right] 0, \bar{\delta}\right]$. Moreover, the final iterate $x_{N(\delta)}$ satisfies the discrepancy principle (1.6) and, for $d_{0}>R \delta$,

$$
N(\delta) \leq\left\lfloor\log _{\eta}\left(R \delta / d_{0}\right)\right\rfloor+1 .^{5}
$$

Proof. We will prove Theorem 4.5 by induction. Therefore, assume that the first $n$ iterates $\left\{x_{0}, \ldots, x_{n}\right\}$ are well defined under the hypotheses of Theorem 4.5 and stay in $B_{\rho}\left(x^{+}\right)$.

If $d_{n} \leq R \delta$, the iteration will be stopped by (1.6) with $N(\delta)=n$. Otherwise, $d_{n}>R \delta$, and we show that the interval determining $\mu_{n}$ is not empty. The bound on $\omega$ implies that the denominator of the lower bound of $R$ is positive. The lower bound on $R$ guarantees that $\tau\left(\omega+(1+\omega) \delta / d_{n}\right)<\tau(\omega+(1+\omega) / R)<\eta-(1+\eta) \omega$.

According to Lemma 2.1, $r_{n}$ and thus the Newton step $s_{n, r_{n}}$ are well defined. By (4.4) and (4.5),

$$
\left\|s_{n, r_{n}}\right\|_{X} \leq 3\left(\frac{C_{K, \kappa} a_{\Theta}\|w\|_{X}}{\Theta \tau_{n}-1}\right)^{1 /(\kappa+1)} \Lambda_{n}^{n /(\kappa+1)} \varepsilon\left(x_{n}, \delta\right)^{-1 /(\kappa+1)} d_{n} .
$$

The lower bound on the $\mu_{k}$ 's yields $\tau_{k} \geq \tau>1, k=0, \ldots, n$ (cf. (4.3)), that is, $\Lambda_{n} \leq \Lambda$. Moreover, $d_{n} / \varepsilon\left(x_{n}, \delta\right) \leq 1 / \omega$. Taking Lemma 4.2 into account, we obtain

$$
\left\|s_{n, i_{n}}\right\|_{X} \leq C_{S}\|w\|_{X}^{1 /(\kappa+1)} d_{0}^{\kappa /(\kappa+1)} \sigma(\kappa)^{n},
$$

where $C_{S}=3\left(C_{K, \kappa} a_{\Theta} /(\Theta \tau-1) / \omega\right)^{1 /(\kappa+1)}$ and

$$
\sigma(\kappa):=\left(\Lambda \eta^{\kappa}\right)^{1 /(\kappa+1)}<1 .^{6}
$$

We define the quantity

$$
\alpha(\delta):=C_{S}\|w\|_{X}^{1 /(\kappa+1)}\left\|F\left(x_{0}\right)-y^{\delta}\right\|_{X}^{\kappa /(\kappa+1)} /(1-\sigma(\kappa)) .
$$

In our formulation of Theorem 4.5 we assumed the product $\|w\|_{X}\left\|F\left(x_{0}\right)-y\right\|_{X}^{\kappa}$ to be sufficiently small. Now we can be more precise: assume that $\|w\|_{X}\left\|F\left(x_{0}\right)-y\right\|_{X}^{\kappa}$ is so small that $\alpha(0)<\rho / 2$. Then there exists a $\bar{\delta}>0$ yielding $\alpha(\delta)<\rho / 2$ for all $\delta \in] 0, \bar{\delta}]$ and the new iterate $x_{n+1}=x_{n}+s_{n, r_{n}}=x_{0}+\sum_{k=0}^{n} s_{k, r_{k}}$ is in $B_{\rho}\left(x^{+}\right)$:

$$
\left\|x^{+}-x_{n+1}\right\|_{X} \leq\left\|x^{+}-x_{0}\right\|_{X}+\sum_{k=0}^{n}\left\|s_{k, r_{k}}\right\|_{X} \leq \rho / 2+\alpha(\delta) \leq \rho .
$$

Further, $d_{n+1} \leq \eta^{n+1} d_{0}$ uniformly in $\left.\left.\delta \in\right] 0, \bar{\delta}\right]$ (Lemma 4.2). This completes the inductive step, thereby finishing the proof of Theorem 4.5.

\footnotetext{
${ }^{5}$ Here, $\lfloor t\rfloor \in \mathbb{Z}$ for $t \in \mathbb{R}$ denotes the greatest integer: $\lfloor t\rfloor \leq t<\lfloor t\rfloor+1$.

${ }^{6}$ Note that $\sigma(\kappa)$ is smaller than 1 since $\kappa>\log _{1 / \eta} \Lambda$.
} 
5. Convergence with rates. Finally, we are able to verify the regularization property of REGINN with conjugate gradients as inner iteration, that is, we will show convergence of $x_{N(\delta)}$ to $x^{+}$as the noise level $\delta$ decreases.

As an additional tool we will use the interpolation inequality (5.1): If $T \in \mathcal{L}(X, Y)$, then

$$
\left\|\left(T^{*} T\right)^{\alpha} x\right\|_{X} \leq\left\|\left(T^{*} T\right)^{\beta} x\right\|_{X}^{\alpha / \beta}\|x\|_{X}^{1-\alpha / \beta} \text { for } 0<\alpha \leq \beta
$$

see, e.g., $[2,12]$.

Under the hypotheses of Theorem 4.5 we have to control the reconstruction error $s_{k}^{\mathrm{e}}=x^{+}-x_{k}$ of the $k$ th iterate, $0 \leq k \leq N(\delta)$ :

$$
\begin{aligned}
\left\|s_{k}^{\mathrm{e}}\right\|_{X}^{2} & =\left\langle\left|A_{k}\right|^{\kappa} s_{k}^{\mathrm{e}},\left|A_{k}\right|^{-\kappa} s_{k}^{\mathrm{e}}\right\rangle_{X} \leq\left\|\left|A_{k}\right|^{\kappa} s_{k}^{\mathrm{e}}\right\|_{X}\left\|\left|A_{k}\right|^{-\kappa} s_{k}^{\mathrm{e}}\right\|_{X} \\
& \stackrel{(5.1)}{\leq}\left\|\left|A_{k}\right| s_{k}^{\mathrm{e}}\right\|_{X}^{\kappa}\left\|s_{k}^{\mathrm{e}}\right\|_{X}^{1-\kappa} C_{K, \kappa} \Lambda^{k}\|w\|_{X},
\end{aligned}
$$

where we also applied (4.5) with $\Lambda_{k} \leq \Lambda$ to obtain the last inequality. Thus,

$$
\begin{aligned}
\left\|s_{k}^{\mathrm{e}}\right\|_{X} & \leq C_{K, \kappa}^{1 /(\kappa+1)} \Lambda^{k /(\kappa+1)}\|w\|_{X}^{1 /(\kappa+1)}\left\|A_{k} s_{k}^{\mathrm{e}}\right\|_{Y}^{\kappa /(\kappa+1)} \\
& \stackrel{(2.2)}{\leq} C_{K, \kappa}^{1 /(\kappa+1)} \Lambda^{k /(\kappa+1)}\|w\|_{X}^{1 /(\kappa+1)}\left(\frac{\left\|y-F\left(x_{k}\right)\right\|_{Y}}{1-C_{Q} \rho}\right)^{\kappa /(\kappa+1)} .
\end{aligned}
$$

Relying on the above estimate, we are now able to copy the proof of Theorem 4.1 from [11] (see also [12, Satz 7.5.17]) to yield the announced convergence result.

THEOREM 5.1. Adopt the assumptions of Theorem 4.5; especially, let the source condition (4.2) be satisfied with $\kappa$ restricted to $\left.] \log _{1 / \eta} \Lambda, 1\right]$. Additionally, assume that $\alpha(0)<\rho / 2$ (see (4.8)), as well as $F\left(x_{0}\right) \neq y=F\left(x^{+}\right)$. Then

$$
\left\|x^{+}-x_{N(\delta)}\right\|_{X} \leq C_{\kappa}\|w\|_{X}^{1 /(\kappa+1)} \delta^{\left(\kappa-\log _{1 / \eta} \Lambda\right) /(\kappa+1)} \quad \text { as } \delta \rightarrow 0,
$$

where $C_{\kappa}$ is a suitable constant.

In the noise-free situation, that is, $\delta=0$, we have that

$$
\left\|x^{+}-x_{k}\right\|_{X}=\mathrm{O}\left(\sigma(\kappa)^{k}\right) \quad \text { as } k \rightarrow \infty
$$

with $\sigma(\kappa)$ from (4.7).

Proof. Plugging $k=N(\delta)$ into (5.2) and taking (1.6) into account give

$$
\left\|s_{N(\delta)}^{\mathrm{e}}\right\|_{X} \leq C_{K, \kappa}^{1 /(\kappa+1)}\|w\|_{X}^{1 /(\kappa+1)}\left(\frac{R+1}{1-C_{Q} \rho}\right)^{\kappa /(\kappa+1)} \Lambda^{N(\delta) /(\kappa+1)} \delta^{\kappa /(\kappa+1)} .
$$

Thus, (5.3) follows from (4.6). Convergence in the noise-free setting is obtained from (5.2) in combination with Lemma 4.2.

6. Computational example. By computational experiments we will demonstrate the increase in numerical efficiency of REGINN when replacing the $\nu$-method by the conjugate gradient iteration as inner iteration. We distinguish the two variants by $\nu$-REGINN and cg-REGINN. Throughout this section let $\nu=1 .^{7}$

\footnotetext{
${ }^{7}$ Any $\nu \geq 1$ is admissible [11, Example 2.1]. However, larger $\nu$ slow down $\nu$-REGINN in the numerical computations presented here.
} 
For our numerical experiments we select a model problem which satisfies our main assumption (2.1). We like to identify the bivariate parameter $c \geq 0$ in the two-dimensional elliptic PDE

$$
\begin{array}{rlrl}
-\Delta u+c u & =f & & \text { in } \Omega, \\
u=g & & \text { on } \partial \Omega
\end{array}
$$

from the knowledge of $u$ in the box $\Omega=] 0,1[2$. In (6.1), $-\Delta$ is the Laplacian. Further, let $f$ and $g$ be continuous functions. If $u$ has no zeros in $\Omega$, then $c$ can be recovered explicitly by $c=(f+\Delta u) / u$. Thus, $c$ is uniquely determined by $u$ but does not depend continuously on it. In the case of noise-corrupted data the inversion formula is useless. Further details about our model problem can be found in Hanke, Neubauer, and Scherzer [6, Example 4.2]. Since we already used our model problem for numerical experiments in $[10,11,12]$ we will be brief in what follows.

We discretize (6.1) by finite differences with respect to the grid points $\left(x_{i}, y_{j}\right)=$ $(i h, j h), 1 \leq i, j \leq m$, where $m \in \mathbb{N}$ and $h=1 /(m+1)$ is the discretization step size. Ordering the grid points lexicographically yields the $m^{2} \times m^{2}$ linear system

$$
(\mathbf{A}+\operatorname{diag}(\mathbf{c})) \mathbf{u}=\mathbf{f},
$$

where $\mathbf{A}$ comes from the difference star of the Laplacian $-\Delta$ and where the components of $\mathbf{c}=\left(\mathbf{c}_{1}, \ldots, \mathbf{c}_{m^{2}}\right)^{t}$ are given by $\mathbf{c}_{\ell(i, j)}=c\left(x_{i}, y_{j}\right)$ with $\ell:\{1, \ldots, m\}^{2} \rightarrow$ $\left\{1, \ldots, m^{2}\right\}$ denoting lexicographical ordering. The boundary values $g$ are incorporated into the right-hand side $\mathbf{f}$. From the convergence theory for finite differences (see, e.g., Hackbusch [3]), we know that the solution $\mathbf{u}$ of the above linear system satisfies $\mathbf{u}_{\ell(i, j)}=u\left(x_{i}, y_{j}\right)+\mathrm{O}\left(h^{2}\right)$ as $h \rightarrow 0$ whenever $u$ is sufficiently smooth.

In this discrete setting we like to reconstruct $\mathbf{c}$ from $\mathbf{u}$. Thus, we have to solve the nonlinear equation

$$
F(\mathbf{c})=\mathbf{u}
$$

with $F: \mathbb{R}_{\geq 0}^{m^{2}} \rightarrow \mathbb{R}^{m^{2}}, F(\mathbf{c})=(\mathbf{A}+\operatorname{diag}(\mathbf{c}))^{-1} \mathbf{f}$. The function $F$ is differentiable with Jacobi-matrix $F^{\prime}(\mathbf{c}) w=-(\mathbf{A}+\operatorname{diag}(\mathbf{c}))^{-1}(F(\mathbf{c}) \odot w)$, where $\odot$ denotes componentwise multiplication of vectors. Moreover, $F^{\prime}$ can be factorized according to (2.1) in a neighborhood of any $\mathbf{c}>0$ (componentwise), where also $F(\mathbf{c})>0 .{ }^{8}$ In our numerical experiments the parameter to be identified is

$$
c^{+}(x, y)=1.5 \sin (2 \pi x) \sin (3 \pi y)+3\left((x-0.5)^{2}+(y-0.5)^{2}\right)+2 .
$$

We have chosen $f$ and $g$ such that $u(x, y)=16 x(x-1) y(1-y)+1$ solves (6.1) with respect to $c^{+}$. As perturbed right-hand side $\mathbf{u}^{\delta}$ for (6.2) we worked with $\mathbf{u}^{\delta}=\widetilde{\mathbf{u}}+\delta \mathbf{v}$, where $\widetilde{\mathbf{u}}_{\ell(i, j)}=u\left(x_{i}, y_{j}\right)$ and $\mathbf{v}=\mathbf{z} /\|\mathbf{z}\|_{h}$. The entries of the random vector $\mathbf{z}$ are uniformly distributed in $[-1,1]$. Therefore, $\left\|\mathbf{u}-\mathbf{u}^{\delta}\right\|_{h} \leq \delta+\mathrm{O}\left(h^{2}\right)$ measured in the weighted Euclidean norm $\|\mathbf{z}\|_{h}=h\left(\sum_{i=0}^{m^{2}} \mathbf{z}_{i}^{2}\right)^{1 / 2}$, which approximates the $L^{2}$-norm over $\Omega$.

In all computations below we started REGINN with initial guess $\mathbf{c}_{0}$, where $\left(\mathbf{c}_{0}\right)_{\ell(i, j)}=$ $c_{0}\left(x_{i}, x_{j}\right)$ and

$$
c_{0}(x, y)=3\left((x-0.5)^{2}+(y-0.5)^{2}\right)+2+128 x(x-1) y(1-y) .
$$

\footnotetext{
${ }^{8}$ The implication $\mathbf{c}>0 \Rightarrow F(\mathbf{c})>0$ holds true, for instance, if $\mathbf{f}>0$ since $\mathbf{A}+\operatorname{diag}(\mathbf{c})$ is an M-matrix whose inverse has only nonnegative entries.
} 


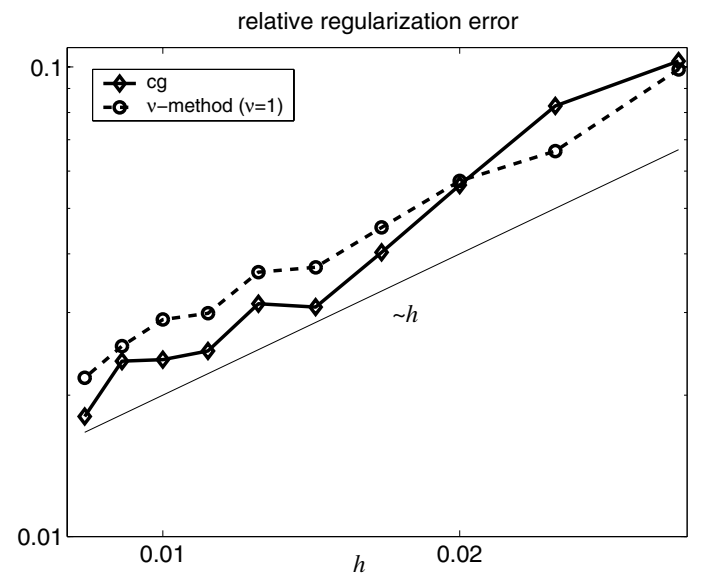

FIG. 6.1. Relative reconstruction error (6.4) of REGINN as a function of $h$ (solid line with $\diamond$ : cg-method as inner iteration; dashed line with $\circ: \nu$-method as inner iteration). The thin solid line indicates decay $\mathrm{O}(h)$ as $h \rightarrow 0$.

Further, we always used $R=1.5$, and we adapted the tolerances $\left\{\mu_{k}\right\}$ in (1.5) dynamically according to scheme (6.3) below, which was proposed in [10, section 6$]$ (see also [12, Kapitel 7.5.3.4]): Initialize $\mu_{\text {start }}=0.1, \gamma=0.9, \mu_{\max }=0.999$, and $\widetilde{\mu}_{0}=\widetilde{\mu}_{1}:=\mu_{\text {start }}$. For $k=0, \ldots, N(\delta)-1$ set

$$
\mu_{k}:=\mu_{\max } \max \left\{R \delta /\left\|F\left(\mathbf{c}_{k}\right)-\mathbf{u}^{\delta}\right\|_{h}, \widetilde{\mu}_{k}\right\},
$$

where $\mathbf{c}_{k}$ is the $k$ th iterate and

$$
\widetilde{\mu}_{k}:=\left\{\begin{array}{ccc}
1-\frac{r_{k-2}}{r_{k-1}}\left(1-\mu_{k-1}\right) & : & r_{k-1} \geq r_{k-2}, \\
\gamma \mu_{k-1} & : & \text { else. }
\end{array}\right.
$$

Figure 6.1 shows relative reconstruction errors by $\nu$-REGINN and cg-REGINN. More precisely, we plotted

$$
\operatorname{err}(h):=\left\|\mathbf{c}_{N(\delta(h))}-\mathbf{c}^{+}\right\|_{h} /\left\|\mathbf{c}^{+}\right\|_{h} \quad \text { with } \delta(h)=10 h^{2}
$$

as a function of $h \in\left\{(10 k)^{-1} \mid k=3, \ldots, 12\right\}$, where $\mathbf{c}_{\ell(i, j)}^{+}=c^{+}\left(x_{i}, y_{j}\right)$ and where $\mathbf{c}_{N(\delta(h))}$ is the output of either $\nu$-REGINN or cg-REGINN. The auxiliary line in Figure 6.1 represents exact decay $\mathrm{O}(h)$ as $h \rightarrow 0$. Thus, our computations indicate that $\operatorname{err}(h)=\mathrm{O}(h)$ as $h \rightarrow 0$. Since $\left\|\mathbf{u}-\mathbf{u}^{\delta(h)}\right\|_{h} \leq \delta(h)+\mathrm{O}\left(h^{2}\right):=\boldsymbol{\delta}(h)=\mathrm{O}\left(h^{2}\right)$ the regularization error achieves maximal order of convergence according to Theorem 5.1, namely, $\operatorname{err}(\boldsymbol{\delta})=\mathrm{O}\left(\boldsymbol{\delta}^{1 / 2}\right)$ as $\boldsymbol{\delta} \rightarrow 0$, that is, $\kappa=1$ and $\Lambda=1$.

Both variants of REGINN deliver errors in comparable magnitude. In this respect there is not much difference between cg-REGINN and $\nu$-REGINN. However, looking at the numerical efficiency we observe a significant difference. In Figure 6.2 we plotted the ratio

$$
q(h):=\frac{\text { cpu-time of } \nu \text {-REGINN to compute } \mathbf{c}_{N(\delta(h))}}{\text { cpu-time of cg-REGINN to compute } \mathbf{c}_{N(\delta(h))}}
$$




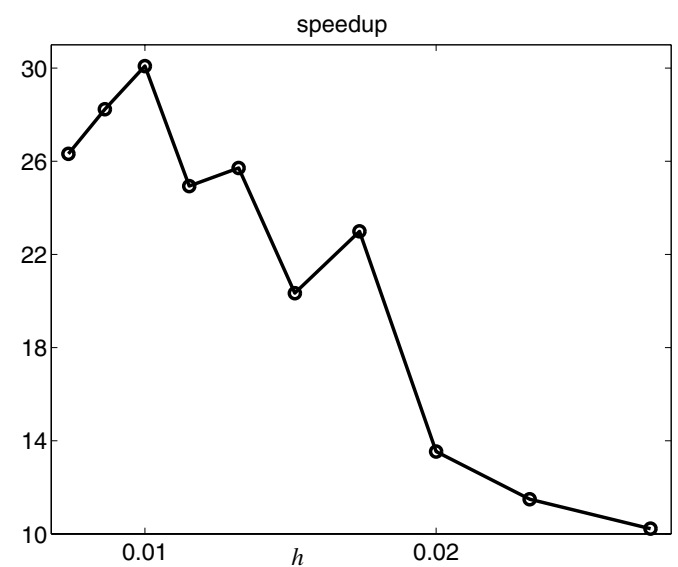

FIG. 6.2. Speedup $q$ (6.5) of $c g$-REGINN over $\nu$-REGINN.

TABLE 6.1

Convergence history of $\nu$-REGINN for $h=0.01, \delta(h)=10 h^{2}$, with respect to the tolerance selection (6.3), where $\mu_{\text {start }}=0.1, \mu_{\max }=0.999$, and $\gamma=0.9$. Overall cpu-time: 240.72 seconds.

\begin{tabular}{c|ccccc}
\hline$k$ & $\mu_{k-1}$ & $r_{k-1}$ & $d_{k} / d_{k-1}$ & $d_{k} /(R \delta)$ & $e_{k}$ \\
\hline 1 & 0.0999 & 40 & 0.2464 & 43.68 & 0.4280 \\
2 & 0.0999 & 75 & 0.1128 & 4.925 & 0.2204 \\
3 & 0.5194 & 99 & 0.5153 & 2.538 & 0.1311 \\
4 & 0.6353 & 105 & 0.6324 & 1.605 & 0.0836 \\
5 & 0.6555 & 176 & 0.6550 & 1.051 & 0.0443 \\
6 & 0.9504 & 242 & 0.9503 & 0.999 & 0.0290 \\
\hline
\end{tabular}

TABLE 6.2

Convergence history of cg-REGINN for $h=0.01, \delta(h)=10 h^{2}$, with respect to the tolerance selection (6.3), where $\mu_{\text {start }}=0.1, \mu_{\max }=0.999$, and $\gamma=0.9$. Overall cpu-time: 8.00 seconds.

\begin{tabular}{c|ccccc}
\hline$k$ & $\mu_{k-1}$ & $r_{k-1}$ & $d_{k} / d_{k-1}$ & $d_{k} /(R \delta)$ & $e_{k}$ \\
\hline 1 & 0.0999 & 1 & 0.3997 & 70.86 & 0.5598 \\
2 & 0.0999 & 1 & 0.1188 & 8.418 & 0.3090 \\
3 & 0.1187 & 6 & 0.1223 & 1.030 & 0.0239 \\
4 & 0.9704 & 1 & 0.9629 & 0.991 & 0.0238 \\
\hline
\end{tabular}

where we did not count cpu-time for preprocessing steps performed by both variants. $^{9}$ Figure 6.2 reveals that cg-REGINN is 10 to 30 times faster than $\nu$-REGINN in our example.

Tables 6.1 and 6.2 record the convergence history of $\nu$-REGINN and cg-REGINN in full detail for the discretization step size $h=0.01$. In both tables

$$
d_{k}:=\left\|F\left(\mathbf{c}_{k}\right)-\mathbf{u}^{\delta(h)}\right\|_{h} \quad \text { and } \quad e_{k}:=\left\|\mathbf{c}_{k}-\mathbf{c}^{+}\right\|_{h} /\left\|\mathbf{c}^{+}\right\|_{h}
$$

denote the nonlinear defect and the relative $L^{2}$-error of the $k$ th iterate, respectively.

Among all Krylov-subspace methods the conjugate gradient iteration is the most efficient one when the discrepancy principle is the used stopping rule; see, e.g., [2,

${ }^{9}$ The experiments were carried out under MATLAB 6.5 on a $2.6 \mathrm{GHz}$ Intel Pentium 4 processor. 
Chapter 7.1] or [12, Kapitel 5.3.6]. As expected, cg-REGINN outperforms $\nu$-REGINN since it takes much fewer inner iterations to yield the correction step which we can observe clearly in the tables (one iteration step of the cg-methods is only slightly more expensive than one iteration step of the $\nu$-method).

7. Concluding remarks. In this paper we proved local convergence with rates for a regularization scheme of inexact Newton type with the cg-method as inner iteration. Theoretical aspects are emphasized; ideas and techniques have been presented to cope with the nonlinearity of the conjugate gradient iteration.

As far as the author knows, the restrictive factorization assumption (2.1) has not been verified for real applications such as, e.g., impedance tomography, ultrasound tomography, and SPECT (single photon emission computed tomography). Therefore the most pressing improvement of the presented analysis is to weaken or to get rid of $(2.1)$.

Nevertheless the practitioner may benefit from our theoretical results in at least two ways: (1) The adaptive tolerance selection scheme (6.3) has a sound justification for cg-REGINN and can be expected to perform well also for more general nonlinearities. (2) A potential convergence analysis of cg-REGINN for a specific application, which does not fall into the general category considered, can be based upon techniques developed here.

Appendix A. Proof of Lemma 3.2. For the sake of simplicity we only prove Lemma 3.2 for a compact operator $T$ (the general result will follow by integration over the spectral family of $T^{*} T$ ). Most of our arguments have been used before by Plato [9, Lemma 5.4] (see also [12, Lemma 5.3.11]) to prove another error estimate for the cg-method.

Let $\left.\left\{\left(\sigma_{j} ; v_{j}, u_{j}\right) \mid j \in \mathbb{N}\right\} \subset\right] 0, \infty[\times X \times Y$ be the singular system of $T$, that is, $T x=\sum_{j=1}^{\infty} \sigma_{j}\left\langle x, v_{j}\right\rangle_{X} u_{j}$ with $\lim _{j \rightarrow \infty} \sigma_{j}=0$ monotonically, and $\left\{v_{j}\right\}$ and $\left\{u_{j}\right\}$ are orthonormal bases in $\mathrm{N}(T)^{\perp}$ and $\overline{\mathrm{R}(T)}$, respectively. ${ }^{10}$

We introduce the spectral family $\left\{\mathcal{E}_{\Lambda}\right\}_{\Lambda>0} \subset \mathcal{L}(X)$ of $T^{*} T$ by ${ }^{11}$

$$
\mathcal{E}_{\Lambda} x:=\sum_{j \in \mathcal{J}(\Lambda)}\left\langle x, v_{j}\right\rangle_{X} v_{j}+P_{\mathrm{N}(T)} x, \quad \mathcal{J}(\Lambda):=\left\{j \in \mathbb{N} \mid \sigma_{j}^{2} \leq \Lambda\right\}
$$

and start with

$$
\left\||T|^{-\nu}\left(\boldsymbol{\xi}_{m}-\boldsymbol{\xi}\right)\right\|_{X} \leq\left\||T|^{-\nu}\left(I-\mathcal{E}_{\Lambda}\right)\left(\boldsymbol{\xi}_{m}-\boldsymbol{\xi}\right)\right\|_{X}+\left\||T|^{-\nu} \mathcal{E}_{\Lambda}\left(\boldsymbol{\xi}_{m}-\boldsymbol{\xi}\right)\right\|_{X} .
$$

We proceed by

$$
\begin{aligned}
\left\||T|^{-\nu}\left(I-\mathcal{E}_{\Lambda}\right)\left(\boldsymbol{\xi}_{m}-\boldsymbol{\xi}\right)\right\|_{X}^{2} & =\sum_{k=1}^{\infty} \sigma_{k}^{-2(\nu+1)}\left|\left\langle|T|\left(I-\mathcal{E}_{\Lambda}\right)\left(\boldsymbol{\xi}_{m}-\boldsymbol{\xi}\right), v_{k}\right\rangle_{X}\right|^{2} \\
& =\sum_{k \notin \mathcal{J}(\Lambda)} \sigma_{k}^{-2(\nu+1)}\left|\left\langle|T|\left(\boldsymbol{\xi}_{m}-\boldsymbol{\xi}\right), v_{k}\right\rangle_{X}\right|^{2} \\
& \leq \Lambda^{-(\nu+1)}\left\|T\left(\boldsymbol{\xi}_{m}-\boldsymbol{\xi}\right)\right\|_{Y}^{2}
\end{aligned}
$$

\footnotetext{
${ }^{10} \mathrm{~N}(B)$ and $\mathrm{R}(B)$ denote the null space and the range of a linear operator, respectively.

${ }^{11} P_{M} \in \mathcal{L}(X)$ denotes the orthogonal projection onto the closed subspace $M$ of $X$.
} 
and

$$
\begin{gathered}
\left\||T|^{-\nu} \mathcal{E}_{\Lambda}\left(\boldsymbol{\xi}_{m}-\boldsymbol{\xi}\right)\right\|_{X} \\
\leq\left\||T|^{-\nu} \mathcal{E}_{\Lambda} p_{m}\left(T^{*} T, \boldsymbol{\eta}\right) \boldsymbol{\xi}\right\|_{X}+\left\||T|^{-\nu} \mathcal{E}_{\Lambda} q_{m-1}\left(T^{*} T, \boldsymbol{\eta}\right) T^{*}(\boldsymbol{\eta}-T \boldsymbol{\xi})\right\|_{X} \\
\leq\left\|\mathcal{E}_{\Lambda}|T|^{\mu-\nu} p_{m}\left(T^{*} T, \boldsymbol{\eta}\right)\right\|_{X}\left\||T|^{-\mu} \boldsymbol{\xi}\right\|_{X} \\
\quad+\left\||T|^{-\nu} \mathcal{E}_{\Lambda} q_{m-1}\left(T^{*} T, \boldsymbol{\eta}\right) T^{*}\right\|_{X}\|\boldsymbol{\eta}-T \boldsymbol{\xi}\|_{X} \\
\leq\left\||T|^{-\mu} \boldsymbol{\xi}\right\|_{X} \sup _{0 \leq \lambda \leq \Lambda} \lambda^{(\mu-\nu) / 2}\left|p_{m}(\lambda, \boldsymbol{\eta})\right| \\
\quad+\left\|\mathcal{E}_{\Lambda} q_{m-1}\left(T^{*} T, \boldsymbol{\eta}\right)|T|^{-\nu} T^{*}\right\|_{X}\|\boldsymbol{\eta}-T \boldsymbol{\xi}\|_{X} .
\end{gathered}
$$

Further,

$$
\begin{aligned}
\| \mathcal{E}_{\Lambda} q_{m-1} & \left(T^{*} T, \boldsymbol{\eta}\right)|T|^{-\nu} T^{*} \|^{2} \\
& =\left\|\mathcal{E}_{\Lambda} q_{m-1}\left(T^{*} T, \boldsymbol{\eta}\right)|T|^{2(1-\nu)} q_{m-1}\left(T^{*} T, \boldsymbol{\eta}\right) \mathcal{E}_{\Lambda}\right\| \\
& \leq\left\|\mathcal{E}_{\Lambda} q_{m-1}\left(T^{*} T, \boldsymbol{\eta}\right)\right\|\left\||T|^{2(1-\nu)} q_{m-1}\left(T^{*} T, \boldsymbol{\eta}\right) \mathcal{E}_{\Lambda}\right\| \\
& \leq \sup _{0 \leq \lambda \leq \Lambda}\left|q_{m-1}(\lambda, \boldsymbol{\eta})\right| \sup _{0 \leq \lambda \leq \Lambda} \lambda^{1-\nu}\left|q_{m-1}(\lambda, \boldsymbol{\eta})\right| .
\end{aligned}
$$

By Lemma 3.1 we have

$$
\sup _{0 \leq \lambda \leq \Lambda}\left|q_{m-1}(\lambda, \boldsymbol{\eta})\right| \leq q_{m-1}(0, \boldsymbol{\eta}) \quad \text { whenever } 0<\Lambda \leq \lambda_{m, 1} .
$$

The representation (3.1) of $p_{m}$ shows that $0 \leq p_{m}(\lambda) \leq 1$ for $\lambda \in\left[0, \lambda_{m, 1}\right]$. Since $p_{m}(\lambda)=1-\lambda q_{m-1}(\lambda)$ we derive that

$$
\begin{aligned}
\sup _{0 \leq \lambda \leq \Lambda} \lambda^{1-\nu}\left|q_{m-1}(\lambda, \boldsymbol{\eta})\right| & \leq\left(\sup _{0 \leq \lambda \leq \Lambda} \lambda\left|q_{m-1}(\lambda, \boldsymbol{\eta})\right|\right)^{1-\nu}\left(\sup _{0 \leq \lambda \leq \Lambda}\left|q_{m-1}(\lambda, \boldsymbol{\eta})\right|\right)^{\nu} \\
& \leq q_{m-1}(0, \boldsymbol{\eta})^{\nu}
\end{aligned}
$$

whenever $0<\Lambda \leq \lambda_{m, 1}$. Finally, for $0<\Lambda \leq \lambda_{m, 1}$, we obtain that

$$
\begin{aligned}
\left\||T|^{-\nu}\left(\boldsymbol{\xi}_{m}-\boldsymbol{\xi}\right)\right\|_{X} \leq \Lambda^{-(\nu+1) / 2}\left\|T\left(\boldsymbol{\xi}_{m}-\boldsymbol{\xi}\right)\right\|_{Y} & +\left\||T|^{-\mu} \boldsymbol{\xi}\right\|_{X} \Lambda^{(\mu-\nu) / 2} \\
& +q_{m-1}(0, \boldsymbol{\eta})^{(\nu+1) / 2}\|\boldsymbol{\eta}-T \boldsymbol{\xi}\|_{X},
\end{aligned}
$$

which yields the stated inequality (3.2) by setting $\Lambda=1 / q_{m-1}(0, \boldsymbol{\eta})$. This choice for $\Lambda$ is admissible since $1 / q_{m-1}(0, \boldsymbol{\eta})<\lambda_{m, 1}$; see Lemma 3.1.

Appendix B. Proof of Lemma 4.3. Before we can prove Lemma 4.3, we need some auxiliary results (Lemmas B.1 and B.2 and Corollary B.3 below). Here we rely on arguments laid out by Plato [9, Kapitel 5] and Nemirovskii [8] (see also [12, Kapitel 5.3]).

Suppose that the first $n$ iterates $\left\{x_{1}, \ldots, x_{n}\right\}$ of REGINN exist and stay in $B_{\rho}\left(x^{+}\right)$. Point of departure is the inequality

$$
\left\|b_{k}^{\varepsilon}-A_{k} s_{k, m}\right\|_{Y} \leq\left\|\mathcal{F}_{\lambda_{m, 1}} \varphi_{m}\left(A_{k} A_{k}^{*}, b_{k}^{\varepsilon}\right) b_{k}\right\|_{Y}+\varepsilon, \quad 1 \leq m \leq \boldsymbol{m}_{\mathrm{T}}
$$

where $0 \leq k \leq n, \boldsymbol{m}_{\mathrm{T}}=\boldsymbol{m}_{\mathrm{T}}(k)$, and $\mathcal{F}_{\Lambda} \in \mathcal{L}(Y), \Lambda>0$, is the orthogonal projection

$$
\mathcal{F}_{\Lambda} y:=\sum_{i \in \mathcal{J}(\Lambda)}\left\langle y, u_{i}\right\rangle_{Y} u_{i}+P_{\mathrm{N}\left(A_{k}^{*}\right)} y
$$


with index set $\mathcal{J}$ as in (A.1). In defining $\mathcal{F}_{\Lambda}$ we used the singular system of $A_{k}{ }^{12}$ Further, the function $\varphi_{m}\left(\cdot, b_{k}^{\varepsilon}\right) \in \mathcal{C}(\mathbb{R})$ in $($ B.1) is

$$
\varphi_{m}\left(\lambda, b_{k}^{\varepsilon}\right):=p_{m}\left(\lambda, b_{k}^{\varepsilon}\right)\left(\frac{\lambda_{m, 1}}{\lambda_{m, 1}-\lambda}\right)^{1 / 2}
$$

where $\lambda_{m, 1}$ is the smallest zero of the $m$ th residual polynomial $p_{m}\left(\cdot, b_{k}^{\varepsilon}\right)$ of the cgmethod with respect to $A_{k}$ and $b_{k}^{\varepsilon}$. A proof of (B.1) is presented, e.g., by Engl, Hanke, and Neubauer [2, Proof of Theorem 7.10].

As $s_{k}^{\mathrm{e}}=x^{+}-x_{k}=s_{0}^{\mathrm{e}}-\sum_{j=0}^{k-1} s_{j, r_{j}}$ we obtain

$$
s_{k}^{\mathrm{e}} \stackrel{(4.2)}{=}|A|^{\kappa} w-\sum_{j=0}^{k-1} A_{j}^{*} q_{r_{j}-1}\left(A_{j} A_{j}^{*}, b_{j}^{\varepsilon}\right) b_{j}^{\varepsilon} .
$$

Note that $s_{k}^{\mathrm{e}} \in \mathrm{D}\left(\left|A_{i}\right|^{-\kappa}\right), i=0, \ldots, n$. Indeed, in using $A_{j}=Q_{j, i} A_{i}$ with $Q_{j, i}=$ $Q\left(x_{j}, x_{i}\right)$ (see $\left.(2.1)\right)$, we obtain that

$$
\begin{aligned}
\left\|\left|A_{i}\right|^{-\kappa} s_{k}^{\mathrm{e}}\right\|_{X} & \leq\left\|\left|A_{i}\right|^{-\kappa}|A|^{\kappa} w\right\|_{X}+\sum_{j=0}^{k-1}\left\|\left|A_{i}\right|^{-\kappa} A_{j}^{*} q_{r_{j}-1}\left(A_{j} A_{j}^{*}, b_{j}^{\varepsilon}\right) b_{j}^{\varepsilon}\right\|_{X} \\
& \stackrel{(2.4)}{\leq} C_{K, \kappa}\|w\|_{X}+\underbrace{\left\|\left|A_{i}\right|^{-\kappa} A_{i}^{*}\right\|}_{=\left\|A_{i}\right\|^{1-\kappa}} \sum_{j=0}^{k-1}\left\|Q_{j, i}^{*} q_{r_{j}-1}\left(A_{j} A_{j}^{*}, b_{j}^{\varepsilon}\right) b_{j}^{\varepsilon}\right\|_{Y} .
\end{aligned}
$$

Thus, by $A_{k} s_{k}^{\mathrm{e}}=b_{k}$,

$$
\begin{aligned}
\left\|\mathcal{F}_{\lambda_{m, 1}} \varphi_{m}\left(A_{k} A_{k}^{*}, b_{k}^{\varepsilon}\right) b_{k}\right\|_{Y} \\
\quad=\left\|\mathcal{F}_{\lambda_{m, 1}} \varphi_{m}\left(A_{k} A_{k}^{*}, b_{k}^{\varepsilon}\right) A_{k}\left|A_{k}\right|^{\kappa}\left|A_{k}\right|^{-\kappa} s_{k}^{\mathrm{e}}\right\|_{Y} \\
\quad \leq \sup _{0 \leq \lambda \leq \lambda_{m, 1}} \lambda^{(\kappa+1) / 2} \varphi_{m}\left(\lambda, b_{k}^{\varepsilon}\right)\left\|\left|A_{k}\right|^{-\kappa} s_{k}^{\mathrm{e}}\right\|_{X} .
\end{aligned}
$$

Techniques from elementary calculus, together with Lemma 3.1, yield

$$
\sup _{0 \leq \lambda \leq \lambda_{m, 1}} \lambda^{(\kappa+1) / 2} \varphi_{m}\left(\lambda, b_{k}^{\varepsilon}\right) \leq 2 q_{m-1}\left(0, b_{k}^{\varepsilon}\right)^{-(\kappa+1) / 2}
$$

for $\kappa \in[0,1]$; see, e.g., $[2,(7.8)]$ or $[12,(5.65)]$. Hence,

$$
\left\|\mathcal{F}_{\lambda_{m, 1}} \varphi_{m}\left(A_{k} A_{k}^{*}, b_{k}^{\varepsilon}\right) b_{k}\right\|_{Y} \leq 2 q_{m-1}\left(0, b_{k}^{\varepsilon}\right)^{-(\kappa+1) / 2}\left\|\left|A_{k}\right|^{-\kappa} s_{k}^{\mathrm{e}}\right\|_{X} .
$$

Finally, (B.1) and (B.4) yield the following lemma.

LEMMA B.1. Let (2.1) hold true and assume that the first $n$ iterates $\left\{x_{1}, \ldots, x_{n}\right\}$ of REGINN exist and stay in $B_{\rho}\left(x^{+}\right)$. If $x_{0} \in B_{\rho}\left(x^{+}\right)$satisfies (4.2), then, for $0 \leq k \leq n$ and $1 \leq m \leq \boldsymbol{m}_{\mathrm{T}}(k)$,

$$
\left\|b_{k}^{\varepsilon}-A_{k} s_{k, m}\right\|_{Y} \leq \varepsilon+2 q_{m-1}\left(0, b_{k}^{\varepsilon}\right)^{-(\kappa+1) / 2}\left\|\left|A_{k}\right|^{-\kappa} s_{k}^{\mathrm{e}}\right\|_{X} .
$$

We need a second auxiliary lemma.

\footnotetext{
${ }^{12}$ More precisely, $\left\{\mathcal{F}_{\Lambda}\right\}_{\Lambda>0}$ is the spectral family of $A_{k} A_{k}^{*}$.
} 
Lemma B.2. Let (2.1) hold true and assume that the first $n$ iterates $\left\{x_{1}, \ldots, x_{n}\right\}$ of REGINN exist and stay in $B_{\rho}\left(x^{+}\right)$. Further, let $x_{0} \in B_{\rho}\left(x^{+}\right)$satisfy (4.2). Choose $\vartheta>2$ and $2<r \leq 2(\vartheta-1)$. Let $0 \leq k \leq n$ and $1 \leq m \leq \boldsymbol{m}_{\mathrm{T}}(k)$.

If $\vartheta q_{m-2}\left(0, b_{k}^{\varepsilon}\right) \leq q_{m-1}\left(0, b_{k}^{\varepsilon}\right)$, then

$$
\frac{r-2}{r-1}\left\|b_{k}^{\varepsilon}-A_{k} s_{k, m-1}\right\|_{Y} \leq \varepsilon+\alpha^{(\kappa+1) / 2} q_{m-1}\left(0, b_{k}^{\varepsilon}\right)^{-(\kappa+1) / 2}\left\|\left|A_{k}\right|^{-\kappa} s_{k}^{\mathrm{e}}\right\|_{X},
$$

where $\alpha=r /\left(1-\vartheta^{-1}\right)$.

Proof. Under our assumptions Plato $[9,(5.16)]$ (see also [12, (5.67)]) established the bound

$$
\frac{r-2}{r-1}\left\|b_{k}^{\varepsilon}-A_{k} s_{k, m-1}\right\|_{Y} \leq\left\|\mathcal{F}_{r \lambda_{m, 1}} p\left(A_{k} A_{k}^{*}\right) b_{k}^{\varepsilon}\right\|_{Y}
$$

with the polynomial $p(\lambda)=p_{m}\left(\lambda, b_{k}^{\varepsilon}\right) /\left(1-\lambda / \lambda_{m, 1}\right)$ of degree $m-1$. The triangle inequality leads to

$$
\left\|\mathcal{F}_{r \lambda_{m, 1}} p\left(A_{k} A_{k}^{*}\right) b_{k}^{\varepsilon}\right\|_{Y} \leq \varepsilon \sup _{0 \leq \lambda \leq r \lambda_{m, 1}}|p(\lambda)|+\left\|\mathcal{F}_{r \lambda_{m, 1}} p\left(A_{k} A_{k}^{*}\right) b_{k}\right\|_{Y} .
$$

To bound $\left\|\mathcal{F}_{r \lambda_{m, 1}} p\left(A_{k} A_{k}^{*}\right) b_{k}\right\|_{Y}$ we are able to apply exactly the same arguments as were used in estimating $\left\|\mathcal{F}_{\lambda_{m, 1}} \varphi_{m}\left(A_{k} A_{k}^{*}\right) b_{k}\right\|_{Y}$; cf. (B.3). Accordingly, if

$$
\sup _{0 \leq \lambda \leq r \lambda_{m, 1}}|p(\lambda)| \leq 1
$$

as well as

$$
\sup _{0 \leq \lambda \leq r \lambda_{m, 1}} \lambda^{(\kappa+1) / 2}|p(\lambda)| \leq \alpha^{(\kappa+1) / 2} q_{m-1}\left(0, b_{k}^{\varepsilon}\right)^{-(\kappa+1) / 2},
$$

then Lemma B.2 is true. Assume, for the moment, that

$$
r \lambda_{m, 1}<2 \lambda_{m, 2} \quad \text { and } \quad \lambda_{m, 1} \leq q_{m-1}\left(0, b_{k}^{\varepsilon}\right)^{-1} /\left(1-\vartheta^{-1}\right)
$$

hold true. The left inequality yields $r \lambda_{m, 1} / \lambda_{m, j}<2, j=2, \ldots, m$, whence

$$
|p(\lambda)|=\prod_{j=2}^{m}\left|1-\lambda / \lambda_{m, j}\right|<1 \quad \text { for } 0<\lambda \leq r \lambda_{m, 1} .
$$

Therefore, (B.6) implies (B.5) and we are left with verifying (B.6).

First we look into the estimate on the right in (B.6). Since the residual polynomials $\left\{p_{m}\left(\cdot, b_{k}^{\varepsilon}\right)\right\}_{1 \leq m \leq m_{\mathrm{T}}}$ are orthogonal, their zeros interlace, that is, $\lambda_{m-1, j}<\lambda_{m, j+1}$, $j=1, \ldots, m-1$. By Lemma 3.1 we therefore have

$$
\begin{aligned}
q_{m-1}\left(0, b_{k}^{\varepsilon}\right) & =\lambda_{m, 1}^{-1}+\sum_{j=1}^{m-1} \lambda_{m, j+1}^{-1} \\
& <\lambda_{m, 1}^{-1}+\sum_{j=1}^{m-1} \lambda_{m-1, j}^{-1}=\lambda_{m, 1}^{-1}+q_{m-2}\left(0, b_{k}^{\varepsilon}\right) .
\end{aligned}
$$


The hypothesis $q_{m-2}\left(0, b_{k}^{\varepsilon}\right) \leq \vartheta^{-1} q_{m-1}\left(0, b_{k}^{\varepsilon}\right)$ implies $q_{m-1}\left(0, b_{k}^{\varepsilon}\right) \leq \lambda_{m, 1}^{-1}+\vartheta^{-1} q_{m-1}\left(0, b_{k}^{\varepsilon}\right)$ and thus the right inequality in (B.6). Since

$$
\begin{gathered}
(\vartheta-1) \lambda_{m-1,1}^{-1} \\
\stackrel{\substack{\text { by } \\
\text { assumpt. }}}{\leq} q_{m-1}\left(0, b_{k}^{\varepsilon}\right)-q_{m-2}\left(0, b_{k}^{\varepsilon}\right) \stackrel{(\text { B.7) }}{<} \lambda_{m, 1}^{-1},
\end{gathered}
$$

we obtain $\lambda_{m, 1}<(\vartheta-1)^{-1} \lambda_{m-1,1}<(\vartheta-1)^{-1} \lambda_{m, 2}$ (interlacing property). In view of $r /(\vartheta-1) \leq 2$ we conclude that the left inequality in (B.6) holds true as well, thereby finishing the proof of Lemma B.2.

Both latter lemmas merge in the next corollary.

Corollary B.3. Let (2.1) hold true and assume the first $n$ iterates $\left\{x_{1}, \ldots, x_{n}\right\}$ of REGINN exist and stay in $B_{\rho}\left(x^{+}\right)$. Further, let $x_{0} \in B_{\rho}\left(x^{+}\right)$satisfy (4.2). Then, to any $\Theta \in] 0,1\left[\right.$, there exists a number $a_{\Theta}$ such that, for $0 \leq k \leq n$,

$$
\Theta\left\|b_{k}^{\varepsilon}-A_{k} s_{k, m-1}\right\|_{Y} \leq \varepsilon+a_{\Theta} q_{m-1}\left(0, b_{k}^{\varepsilon}\right)^{-(\kappa+1) / 2}\left\|\left|A_{k}\right|^{-\kappa} s_{k}^{\mathrm{e}}\right\|_{X},
$$

where $1 \leq m \leq \boldsymbol{m}_{\mathrm{T}}(k)$. The number $a_{\Theta}$ only depends on $\Theta$ and $\kappa$.

Proof. There is exactly one $r=r(\Theta)>2$ such that $\Theta=\frac{r-2}{r-1}$. Let this $r$ be fixed and define $\vartheta=r / 2+1>2$, that is, $r=2(\vartheta-1)$. Exactly one of the following two cases holds true.

1. In the case of $\vartheta q_{m-2}\left(0, b_{k}^{\varepsilon}\right) \leq q_{m-1}\left(0, b_{k}^{\varepsilon}\right)$ the assertion follows immediately from Lemma B.2 when setting

$$
a_{\Theta, 1}:=\left(\frac{r}{1-\vartheta^{-1}}\right)^{(\kappa+1) / 2}=\left(\frac{r}{1-(r / 2+1)^{-1}}\right)^{(\kappa+1) / 2} .
$$

2. In the case of $q_{m-1}\left(0, b_{k}^{\varepsilon}\right)<\vartheta q_{m-2}\left(0, b_{k}^{\varepsilon}\right)$ we argue with Lemma B.1. Since $\Theta<1$ and $q_{m-2}\left(0, b_{k}^{\varepsilon}\right)^{-1}<\vartheta q_{m-1}\left(0, b_{k}^{\varepsilon}\right)^{-1}$ we have

$$
\begin{aligned}
\Theta\left\|b_{k}^{\varepsilon}-A s_{k, m-1}\right\|_{Y} & \leq \varepsilon+2 q_{m-2}\left(0, b_{k}^{\varepsilon}\right)^{-(\kappa+1) / 2}\left\|\left|A_{k}\right|^{-\kappa} s_{k}^{\mathrm{e}}\right\|_{X} \\
& <\varepsilon+a_{\Theta, 2} q_{m-1}\left(0, b_{k}^{\varepsilon}\right)^{-(\kappa+1) / 2}\left\|\left|A_{k}\right|^{-\kappa} s_{k}^{\mathrm{e}}\right\|_{X}
\end{aligned}
$$

with

$$
a_{\Theta, 2}:=2 \vartheta^{(\kappa+1) / 2}=2(r / 2+1)^{(\kappa+1) / 2} .
$$

So, the assertion of Corollary B.3 is verified with $a_{\Theta}=\max \left\{a_{\Theta, 1}, a_{\Theta, 2}\right\}$.

Finally we are in a position to verify Lemma 4.3: The $\mu_{k}$ 's and $R$ satisfy the requirements of Lemma 2.1. Hence, $\tau_{k}>1$ (see (4.3)), and $\tau_{k} \varepsilon \leq\left\|b_{k}^{\varepsilon}-A_{k} s_{k, r_{k}-1}\right\|_{Y}$ (see $(1.5))$. Since $1 \leq r_{k} \leq \boldsymbol{m}_{\mathrm{T}}(k)$ we obtain

$$
\Theta \tau_{k} \varepsilon \leq \Theta\left\|b_{k}^{\varepsilon}-A_{k} s_{k, r_{k}-1}\right\|_{Y} \leq \varepsilon+a_{\Theta} q_{r_{k}-1}\left(0, b_{k}^{\varepsilon}\right)^{-(\kappa+1) / 2}\left\|\left|A_{k}\right|^{-\kappa} s_{k}^{\mathrm{e}}\right\|_{X}
$$

by Corollary B.3. A simple rearrangement of terms yields the assertion of Lemma 4.3.

\section{REFERENCES}

[1] A. B. BAKushinskiI, The problem of the convergence of the iteratively regularized GaussNewton method, Comput. Math. Phys., 32 (1992), pp. 1353-1359. 
[2] H. W. Engl, M. Hanke, and A. Neubauer, Regularization of Inverse Problems, Math. Appl. 375, Kluwer Academic, Dordrecht, The Netherlands, 1996.

[3] W. Hackbusch, Elliptic Differential Equations: Theory and Numerical Treatment, Springer Ser. Comput. Math. 18, Springer-Verlag, Heidelberg, 1992.

[4] M. Hanke, A regularizing Levenberg-Marquardt scheme, with applications to inverse groundwater filtration problems, Inverse Problems, 13 (1997), pp. 79-95.

[5] M. HANke, Regularizing properties of a truncated Newton-CG algorithm for non-linear inverse problems, Numer. Funct. Anal. Optim., 18 (1997), pp. 971-993.

[6] M. Hanke, A. Neubauer, And O. Scherzer, A convergence analysis of the Landweber iteration for nonlinear ill-posed problems, Numer. Math., 72 (1995), pp. 21-37.

[7] B. Kaltenbacher, A posteriori parameter choice strategies for some Newton type methods for the regularization of nonlinear ill-posed problems, Numer. Math., 79 (1998), pp. 501-528.

[8] A. S. NemirovskiI, The regularizing properties of the adjoint gradient method in ill-posed problems, USSR Comp. Math. Math. Phys., 26 (1986), pp. 7-16.

[9] R. Plato, Über die Diskretisierung und Regularisierung schlecht gestellter Probleme, Ph.D. thesis, Fachbereich Mathematik der Technischen Universität Berlin, Berlin, 1990. Available online from www.math.tu-berlin.de/ plato/promo.ps.

[10] A. RIEDER, On the regularization of nonlinear ill-posed problems via inexact Newton iterations, Inverse Problems, 15 (1999), pp. 309-327.

[11] A. Rieder, On convergence rates of inexact Newton regularizations, Numer. Math., 88 (2001), pp. 347-365.

[12] A. Rieder, Keine Probleme mit Inversen Problemen, Vieweg, Wiesbaden, Germany, 2003.

[13] E. Zeidler, Nonlinear Functional Analysis and Its Applications I: Fixed-Point Theorems, Springer-Verlag, New York, 1993. 